\title{
Image Transmission using M-QAM OFDM System over Composite Fading Channel
}

\author{
Ashu Tuli ${ }^{1}$, Narender Kumar ${ }^{2}$ Kanchan Sharma ${ }^{3}$ \\ (GGSIPU, New Delhi, India)
}

\begin{abstract}
This paper is focused on the transmission of images using M-QAM OFDM system over Composite Fading channel. Recently proposed Generalized K Fading channel is very versatile channel which accurately approximates many of the commonly used channel models such as Nakagami-m, Log-Normal distributions and the mixture of the two distributions for the composite fading. The robustness of multiple access schemes such as Orthogonal Frequency Division Multiplexing (OFDM) for the wireless communication system is well proven, but the introduction of M-QAM with OFDM has led to good tradeoff between throughput, symbol-error robustness and a reduction of the peak-to-average ratio of the OFDM signal. The results show that the Generalized K Fading channel is highly efficient for predicting the performance of fading and shadowing and demonstrating their effects simultaneously.
\end{abstract}

Keywords: Orthogonal Frequency Division Multiplexing, M-Quadrature Amplitude Modulation, Generalized K Fading Channel, Bit Error Rate

\section{Introduction}

In recent times world research is mainly focused on Wireless Communication. The rapid growth of the use of Bluetooth, Android and iOS based mobile systems, Blackberry Services and Wi-Fi are creating fast and effective changes in the way people communicate. The requirement of high date rate applications in daily life has shifted focus on quality transmission of visual data such as images over the Wireless Medium. To provide such quality transmission, wireless is convenient, but is not perfect. There are limitation and difficulties such as bandwidth limitations, signal attenuation, co-channel interferences, and time varying channel. Consequently, the quality of transmitted images gets degraded. The received signal over a wireless channel is usually characterized by the joint effect of two independent random processes such as small scale fading due to reflected and scattered signal or due to shadowing from various obstacles in the propagation path. Several statistical models are developed to study the effect of fading and shadowing individually, but since fading and shadowing happens simultaneously, it is necessary to have composite models which can represent their effects simultaneously.

Recent work related to this topic is described as follows.

Rayleigh and Rician fading distributions in frequency selective fading channels have already been deployed and studied in depth for OFDM systems [1,2]. Various channel estimation and diversity schemes have been proposed in literature to enhance the error performance under Rayleigh and Rician fading channel [3-5]. Nakagami-m fading distribution is another useful and important model to characterize the fading channel [6]. Kang et al. [7] modeled the OFDM- BPSK system with frequency selective fading channel. Zheng et al. [8] further enhanced the work by presenting asymptotic BER performance of OFDM system in frequency selective Nakagami-m channel. In [9], accurate error performance of OFDM systems is analyzed on the basis of number of channel taps in Nakagami m fading environment. In [4], BER performance of OFDM is analyzed on the basis of BPSK and QPSK in Generalized Gamma Fading Channel.

This paper focus on investigating the performance of fading and shadowing for the grayscale image transmission using the M- QAM OFDM system over a Generalized K Fading channel. To the best of our information, the performance of fading and shadowing over this channel has not been investigated so far for image transmission. By comparing the transmission of images using different fading and shadowing parameters, it has been observed that these parameters affect the quality of image drastically.

The paper is organized as follows. Section II, briefly review M-QAM, OFDM system and Generalized K Fading channel. Section III describes the system parameters used for image transmission in the present work. Section IV presents experimental results and their discussion. Section V provides the Conclusion and References

\subsection{M-Quadrature Amplitude Modulation}

\section{Background}

QAM is the encoding of the information into a carrier wave by variation of the amplitude of both the carrier wave and a "quadrature ee carrier that is $90^{\circ}$ out of phase with the main carrier in accordance with two 
input signals. That is, the amplitude and the phase of the carrier wave are simultaneously changed according to the information you want to transmit. M- Quadrature Amplitude Modulation (M-QAM) is a widely used modulation technique that provides high transmission rates, high bandwidth and high energy efficiency M-QAM has constellation size as $\mathrm{M}=2^{\mathrm{n}}$ for $\mathrm{n}$ a positive integer. In 16 Quadrature Amplitude Modulation (16-QAM), there are 16 combination of real and imaginary values. This results in a total of 16 possible states for the signal. Figure. 1 depicts the Signal Constellation for 16-QAM.

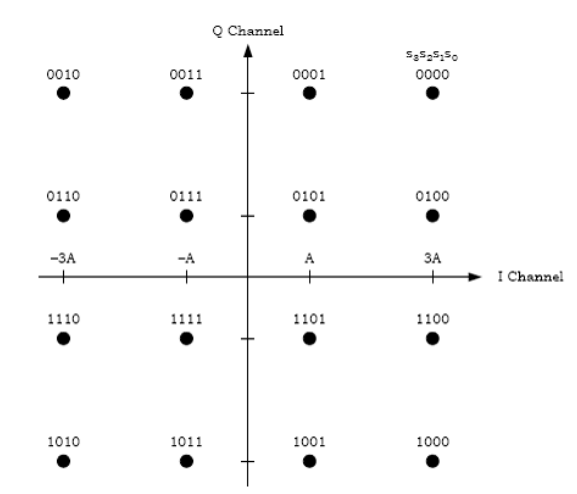

Fig. 1: Signal Constellation for 16- QAM Modulation

It can transition from any state to any other state at every symbol time. Since $16=2^{4}$, four bits per symbol can be sent which consists of two bits for I and two bits for Q. The symbol rate is one fourth of the bit rate. So 16-QAM modulation format produces a more spectrally efficient transmission [14].

\subsection{Generalized K Fading Channel}

Short Term Fading refers to large changes in the amplitude and phase of a signal caused by a small change in the position of the transmitter or receiver. It is generally represented using Nakagami-m distribution as it corresponds to the results of short wave ionospheric propagation. The envelope of the transmitted signal over the Nakagami fading, $X$, has the probability density function (pdf) $\rho_{X}(\mathrm{x})$ given as follows

$$
\rho_{\mathrm{X}}(\mathrm{x})=\frac{2}{\Gamma\left(\mathrm{m}_{\mathrm{m}}\right)}\left(\frac{\mathrm{m}_{\mathrm{m}}}{\Omega}\right)^{\mathrm{m}_{\mathrm{m}}} \mathrm{x}^{2 \mathrm{~m}_{\mathrm{m}}-1} \mathrm{e}^{-\frac{\mathrm{m}_{\mathrm{m}}}{\Omega} \mathrm{x}^{2}}, \mathrm{x}>0
$$

Where, $m_{m}$ is the fading severity parameter $\Omega(\Omega=\overline{\mathrm{X}})$ is the average received power and $\Gamma($.$) is the$ Gamma Function. In terrestrial and satellite land-mobile systems, the signal quality is also affected by slow variation of the mean signal level due to the shadowing from terrain, buildings and trees. Consequently, multipath fading is superimposed on shadowing. Hence, the pdf of the envelope of the received signal $\rho_{X}(x)$ will be conditioned on the average power $\Omega$ leading to Equation (2).

$$
\rho_{\mathrm{x} / \Omega}(\mathrm{x} / \mathrm{y})=\frac{2}{\Gamma\left(\mathrm{m}_{\mathrm{m}}\right)}\left(\frac{\mathrm{m}_{\mathrm{m}}}{\Omega}\right)^{\mathrm{m}_{\mathrm{m}}} \mathrm{x}^{2 \mathrm{~m}_{\mathrm{m}}-1} \mathrm{e}^{-\frac{\mathrm{m}_{\mathrm{m}}}{\mathrm{y}} \mathrm{x}^{2}}, \mathrm{x}>0
$$

Therefore in combined multipath and shadowing fading, equation (1) can be expressed as follows

$$
\mathrm{p}_{\mathrm{X}}(\mathrm{x})=\int_{0}^{+\infty} \mathrm{p}_{\mathrm{X} / \Omega}(\mathrm{x} / \mathrm{y}) \mathrm{p}_{\Omega}(\mathrm{y}) \mathrm{dy}, \mathrm{x}>0
$$

Where, $p_{\Omega}(y)$ is the pdf of the average power which is usually modeled by the Log-Normal distribution. In order to mitigate difficulty to derive close form of some metric performance measure, using the moment matching method, it was argued that a gamma shadowing is an excellent match to the lognormal shadowing seen in wireless systems [20], so Gamma distribution used to model the average power random variations given as follows

$$
\mathrm{p}_{\Omega}(\mathrm{y})=\frac{1}{\Gamma\left(\mathrm{m}_{\mathrm{m}}\right)}\left(\frac{\mathrm{m}_{\mathrm{s}}}{\Omega_{0}}\right)^{\frac{\mathrm{k}+\mathrm{m}}{2}} \mathrm{~K}_{\mathrm{k}-\mathrm{m}}\left[2\left(\frac{\mathrm{m}}{\Omega_{0}}\right)^{1 / 2} \mathrm{x}\right], \mathrm{x}>0
$$

Where, $\mathrm{m}_{\mathrm{s}}>0$ reflects the shadowing severity and $\Omega_{0}$ is the local mean power [11]. Therefore, the pdf of the received signal envelope due to composite fading (shadowing and multipath) comprise to the Generalized- $\mathrm{K}$ distribution $\left(\mathrm{K}_{\mathrm{G}}\right)$ which is given by, 


$$
f_{X}(x)=\frac{4 x^{m+k-1}}{\Gamma(m) \Gamma(k)}\left(\frac{m}{\Omega_{0}}\right)^{\frac{k+m}{2}} K_{k-m}\left[2\left(\frac{m}{\Omega_{0}}\right)^{1 / 2} x\right], x>0
$$

Where, $\mathrm{k}$ and $\mathrm{m}$ are the shaping parameter of the [17] distribution. The parameter $\mathrm{m}$ is a fading severity parameter and $\mathrm{k}$ is a shadowing severity parameter. The two shaping parameters, $\mathrm{m}$ and $\mathrm{k}$, can take different values which approximate several shadowing conditions, from severe shadowing $(\mathrm{k} \rightarrow 0)$ to no shadowing $(\mathrm{k} \rightarrow \infty)$. This model assumes a Nakagami or Rayleigh density function for the envelope of the received signal and the power of the envelope [21] has a gamma distribution. This composite model can be used to analyze the performance of wireless systems in shadowed fading channels [12]. Since $\mathrm{K}_{\mathrm{G}}$ is a two parameter distribution, various fading and shadowing models can be described by using different value combinations for $\mathrm{k}$ and/or $\mathrm{m}$. For example, as $\mathrm{k} \rightarrow \infty$, it approximates the [18 ]well-known Nakagami- $\mathrm{m}$ distribution ; for $\mathrm{m}=1$, it coincides with the K distribution and approximately models Rayleigh-Lognormal (R-L) fading conditions, while for $\mathrm{m} \rightarrow \infty$ and $\mathrm{k} \rightarrow \infty$, approaches the additive white Gaussian noise (AWGN) channel [4].

\subsection{Orthogonal Frequency Division Multiplexing}

The OFDM is a recently used technology in the broadband communication like IEEE 802.11 wireless local area networks (WLANs) and IEEE 802.16 broadband fixed wireless access networks in a wireless multipath environment. The orthogonal frequency division multiplexing (OFDM) is a popular multi-carrier transmission scheme which optimizes overall channel capacity and improves throughput. The basic principle of OFDM is to split a higher rate data stream into a number of lower rate data streams which are transmitted simultaneously over a number of sub- carriers. The dispersion in time caused by the multipath delay spread is decreased relatively due to the increase of the symbol duration for lower rate parallel sub-carriers. Inter-symbol interference (ISI) is completely eliminated by the addition of the guard time in every OFDM symbol [10]. Figure. 2 depicts the block diagram of the OFDM system used to transmit a gray scale image over Generalized $\mathrm{K}$ Fading channel. The OFDM system parameters used in this simulation are mentioned in Table 1

Table 1: OFDM Parameters

\begin{tabular}{|l|l|}
\hline Parameters & Value \\
\hline FFT/IFFT Size & 64 \\
\hline No of Data Subcarriers & 48 \\
\hline No of Pilot Subcarriers & 4 \\
\hline No of Total Subcarriers & 52 \\
\hline SNR_dB & $0: 2: 20$ \\
\hline Modulation Type & M-QAM \\
\hline Constellation & $4,8,16,32,64,128,256$ \\
\hline Channel & Generalized K Fading channel \\
\hline Guard Type & Cyclic Prefix \\
\hline
\end{tabular}

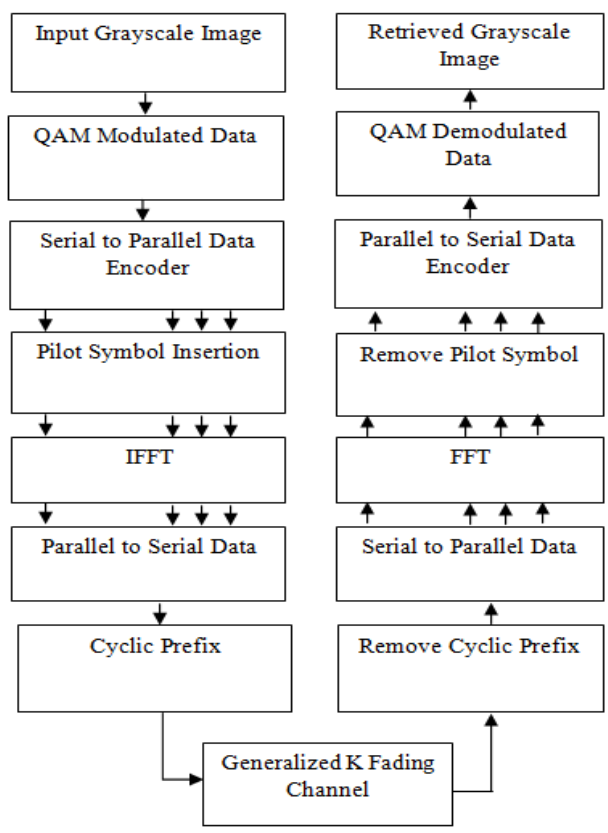

Fig. 2: Block diagram of grayscale image communication using a M-QAM OFDM system 


\section{System Description}

Figure. 3 depicts the sequence of events used in the present work to transmit three different gray scale images of size $200 * 320$ over Generalized K Fading channel. These images are Clown, Wheel and Cameraman and are depicted in Figures. 4-6 respectively. The OFDM system parameters used in this simulation are tabulated in Table 1 . These images are firstly reshaped according to M-QAM value, pass through an OFDM modulator block and then transmitted over the said Generalized K Fading channel. Table 2 comprises of the different fading and shadowing parameters used in the present work.

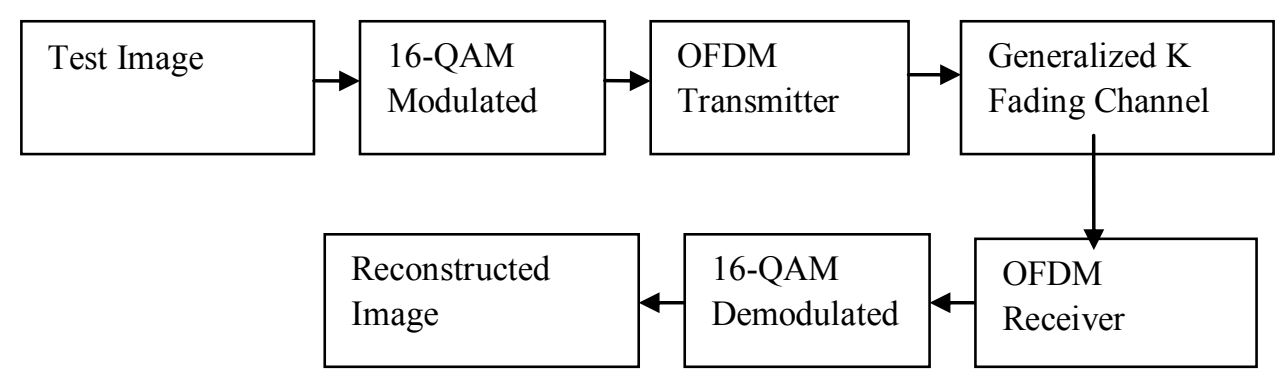

Fig. 3: Block diagram of system description

Table 2: Fading Parameter (m) and Shadowing Parameters (k)

\begin{tabular}{|l|l|}
\hline Fading Parameter (m) & $1,2,3,4,5$ \\
\hline Shadowing Parameter (k) & $1,2,3,4,5$ \\
\hline
\end{tabular}

These images are then received and pass through an OFDM demodulator block before being decoded. The performance is analyzed using BER (Bit Error Rate) which is defined as the number of errors in a given number of transmitted bits over a communication channel that have been changed due to noise, interference or distortion[13].

\section{Experimental Results And Discussion}

Figures. $4-6$ depicts original gray scale images Cameraman, Clown and Wheel each of size 200*320. However in order to adjust these within the manuscript, the size is reduced to half. Figures. 7-9 depict the respective reconstructed images at an $\mathrm{Eb} / \mathrm{No}=12 \mathrm{~dB}, \mathrm{QAM}=16, \mathrm{~m}=1$ and $\mathrm{k}=1$. The channel BER values of reconstructed images with maximum fading and shadowing are tabulated in Table 3 . We observe that for all these images, the low channel BER is achieved at lower Eb/No.

Another significant observation is that as we increase the value of fading and shadowing parameters, the channel starts behaving as AWGN channel and the probability of error between transmitted and reconstructed image data decreases, thereby giving better quality of reconstructed images. The channel BER values of reconstructed images with minimum fading and shadowing are tabulated in Table 4. Although, we have carried out our simulation for three different grayscale images, the plots channel BER vs Eb/No are shown in the case of Clown image in this paper. This is because the results obtained in the case of clown are the best. It has been observed that the behavior of the respective plots for all three images is identical

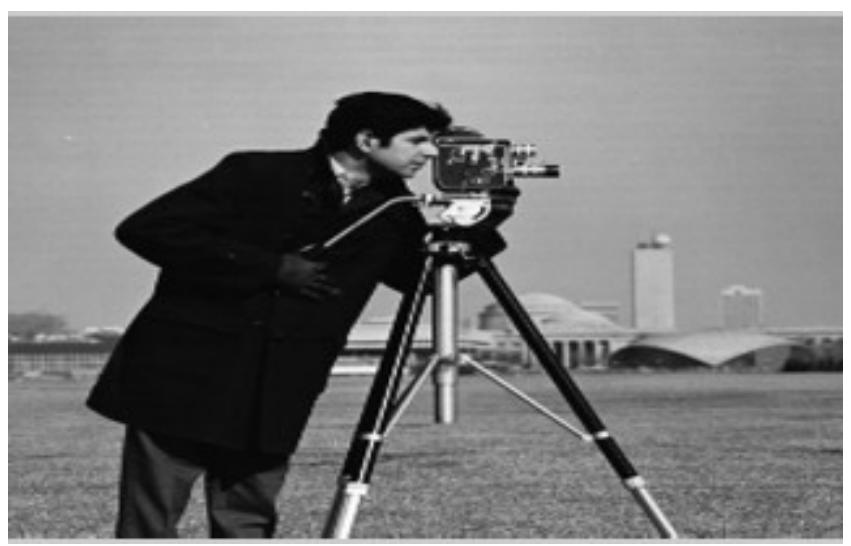

Fig. 4: Original grayscale image Cameraman 


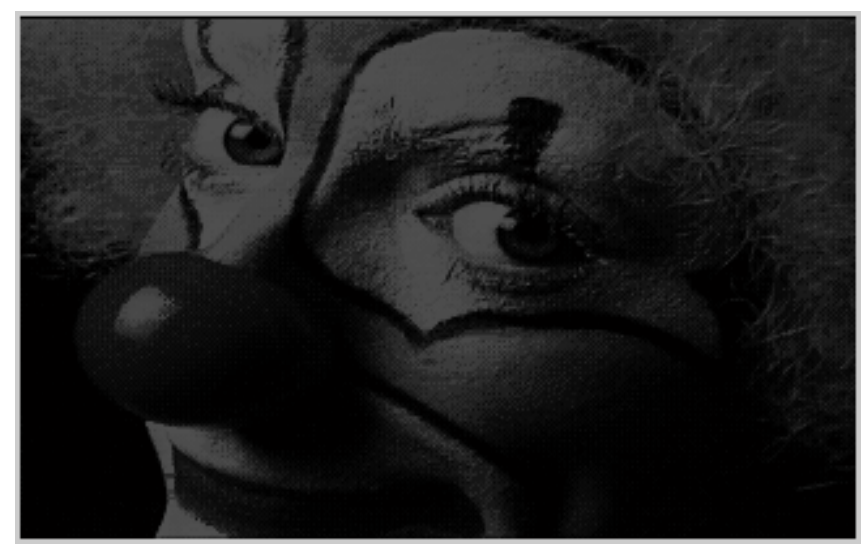

Fig. 5: Original grayscale image Clown

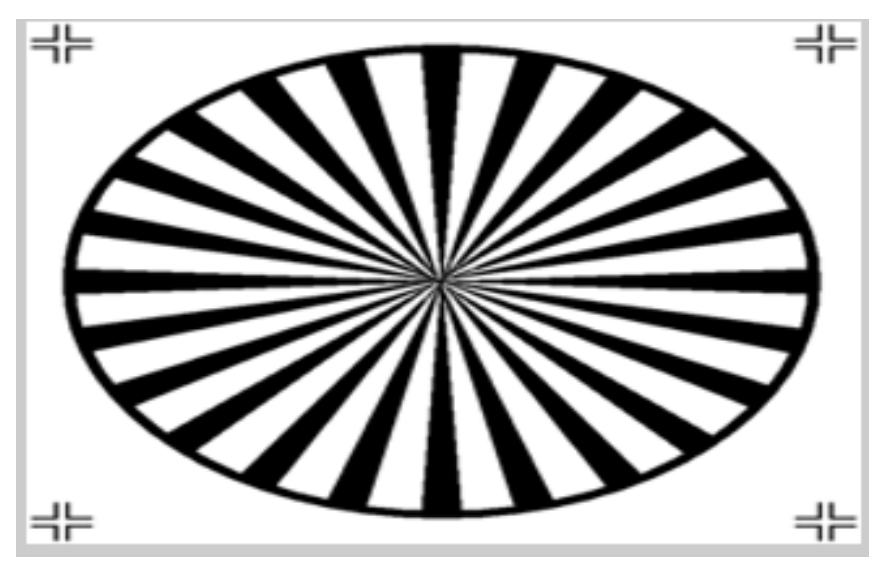

Fig. 6: Original grayscale image Wheel

Figure. 10 shows the plot of channel BER vs Eb/No for the different values of fading parameter (m) when shadowing parameter $(\mathrm{k})$ has been kept constant as maximum $(\mathrm{k}=1)$. In this case higher the size of the fading, the higher is the channel BER achieved at lower Eb/No. It has been noticed that when $\mathrm{m}$ becomes larger, better performance is obtained because the larger $m$ represents the less severe fading

Figure. 11 shows the plot of channel BER vs Eb/No for the different values of shadowing parameter (k) when fading parameter $(\mathrm{m})$ has been kept constant as maximum $(\mathrm{m}=1)$. In this case higher the size of the shadowing, the higher is the channel BER achieved at lower Eb/No. It has been noticed that when $\mathrm{k}$ becomes larger, the plots of BER moves towards lower values hinting that the amount of fading will be higher.

Channel Eb/No $=12 \mathrm{~dB}, \mathrm{QAM}=16, \mathrm{~m}=1, \mathrm{k}=1$

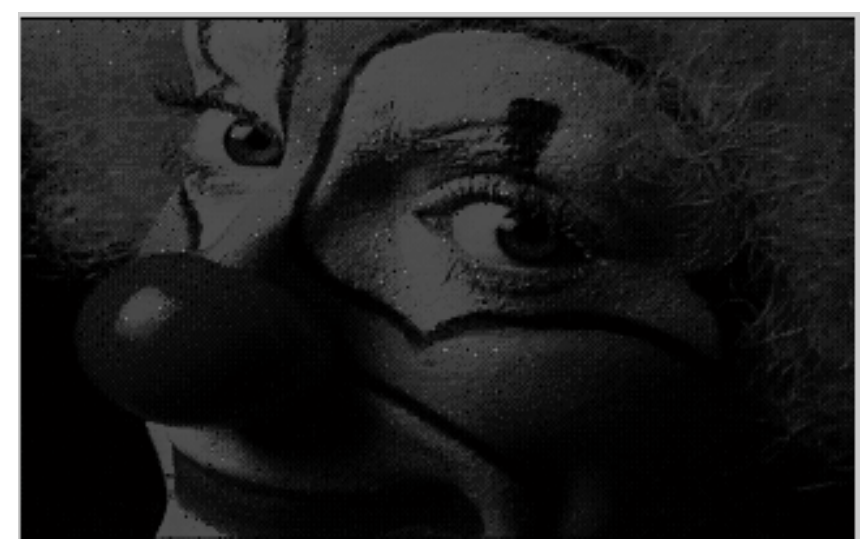

Fig. 7: Reconstructed grayscale image Clown

Channel $\mathrm{Eb} / \mathrm{No}=12 \mathrm{~dB}, \mathrm{QAM}=16, \mathrm{~m}=1, \mathrm{k}=1$ 


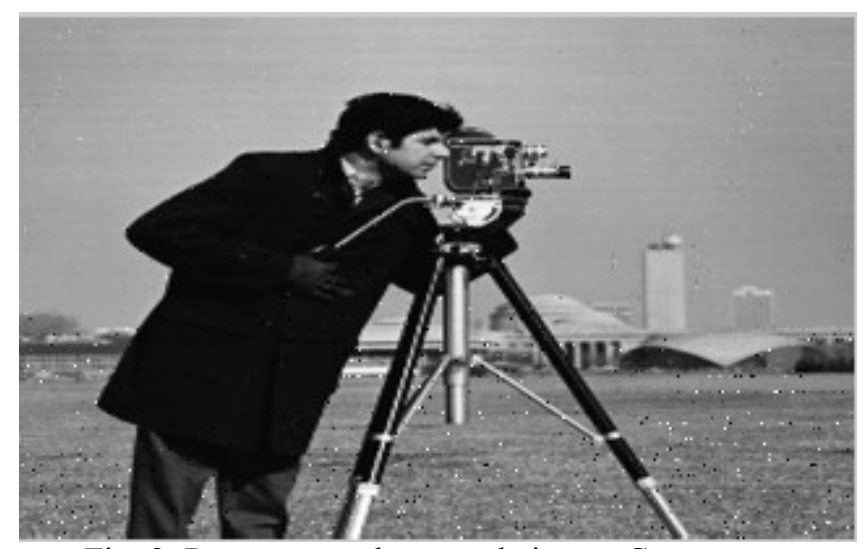

Fig. 8: Reconstructed grayscale image Cameraman

Channel $\mathrm{Eb} / \mathrm{No}=12 \mathrm{~dB}, \mathrm{QAM}=16, \mathrm{~m}=1, \mathrm{k}=1$

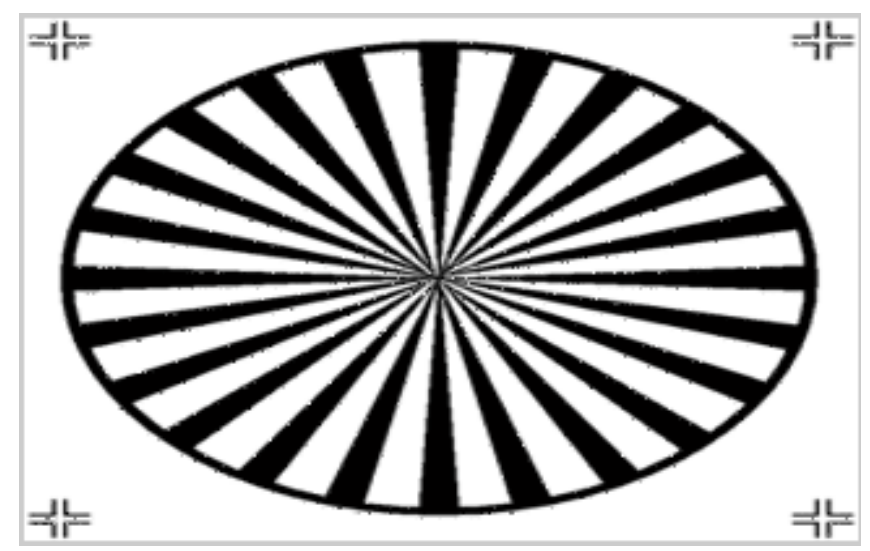

Fig. 9: Reconstructed grayscale image Wheel Channel $\mathrm{Eb} / \mathrm{No}=12 \mathrm{~dB}, \mathrm{QAM}=16, \mathrm{~m}=1, \mathrm{k}=1$

Table 3: Channel BER, for Cameraman, Clown and Wheel w. r. t Channel Eb/No with maximum fading and Shadowing in QAM Modulated OFDM system

\begin{tabular}{|c|c|c|c|}
\hline \multicolumn{4}{|c|}{ Bit Error Rate } \\
\hline \multicolumn{4}{|c|}{$\mathrm{QAM}=16, \mathrm{~m}=1, \mathrm{k}=1$} \\
\hline Eb/No & Cameraman & Clown & Wheel \\
\hline 2 & 0.0593 & 0.0529 & 0.0314 \\
\hline 3 & 0.0486 & 0.0429 & 0.0249 \\
\hline 4 & 0.0391 & 0.0351 & 0.0194 \\
\hline 5 & 0.0323 & 0.0286 & 0.0158 \\
\hline 6 & 0.0258 & 0.0234 & 0.0125 \\
\hline 7 & 0.0208 & 0.0186 & 0.0101 \\
\hline 8 & 0.0163 & 0.0148 & 0.0082 \\
\hline 9 & 0.0123 & 0.0111 & 0.0064 \\
\hline 10 & 0.0088 & 0.0081 & 0.0047 \\
\hline 11 & 0.0061 & 0.0058 & 0.0034 \\
\hline 12 & 0.0039 & 0.0037 & 0.0023 \\
\hline 13 & 0.0021 & 0.0021 & 0.0014 \\
\hline 14 & 0.0011 & 0.0011 & 0.0008 \\
\hline 15 & 0.0004 & 0.0005 & 0.0004 \\
\hline 16 & 0.0002 & 0.0002 & 0.0001 \\
\hline 17 & 0 & 0.0001 & 0.0001 \\
\hline 18 & 0 & 0 & 0 \\
\hline 19 & 0 & 0 & 0 \\
\hline 20 & 0 & 0 & 0 \\
\hline
\end{tabular}


Table 4: Channel BER, for Cameraman, Clown and Wheel w. r. t Channel Eb/No with minimum fading and Shadowing in QAM Modulated OFDM system

\begin{tabular}{|c|c|c|c|}
\hline \multicolumn{4}{|c|}{ Bit Error Rate } \\
\hline \multicolumn{4}{|c|}{$\mathrm{QAM}=16, \mathrm{~m}=5, \mathrm{k}=5$} \\
\hline Eb/No & Cameraman & Clown & Wheel \\
\hline 2 & 0.0756 & 0.0728 & 0.0589 \\
\hline 3 & 0.0583 & 0.0557 & 0.0439 \\
\hline 4 & 0.0424 & 0.0406 & 0.0321 \\
\hline 5 & 0.0297 & 0.0284 & 0.0217 \\
\hline 6 & 0.0194 & 0.0185 & 0.0139 \\
\hline 7 & 0.0122 & 0.0115 & 0.0083 \\
\hline 8 & 0.0073 & 0.0068 & 0.0049 \\
\hline 9 & 0.0041 & 0.0039 & 0.0027 \\
\hline 10 & 0.0019 & 0.0019 & 0.0014 \\
\hline 11 & 0.0009 & 0.0009 & 0.0007 \\
\hline 12 & 0.0003 & 0.0003 & 0.0003 \\
\hline 13 & 0.0001 & 0.0001 & 0.0001 \\
\hline 14 & 0 & 0 & 0 \\
\hline 15 & 0 & 0 & 0 \\
\hline 16 & 0 & 0 & 0 \\
\hline 17 & 0 & 0 & 0 \\
\hline 18 & 0 & 0 & 0 \\
\hline 19 & 0 & 0 & 0 \\
\hline 20 & 0 & 0 & 0 \\
\hline
\end{tabular}

Figure. 12 shows the plot of channel BER vs Eb/No for the different values of $M$ in M-QAM with maximum fading parameter and shadowing parameter $(\mathrm{m}=1, \mathrm{k}=1)$. In this case it has been analyzed that with higher-order QAM, the BER performance is the worst and large amount of information is transmitted

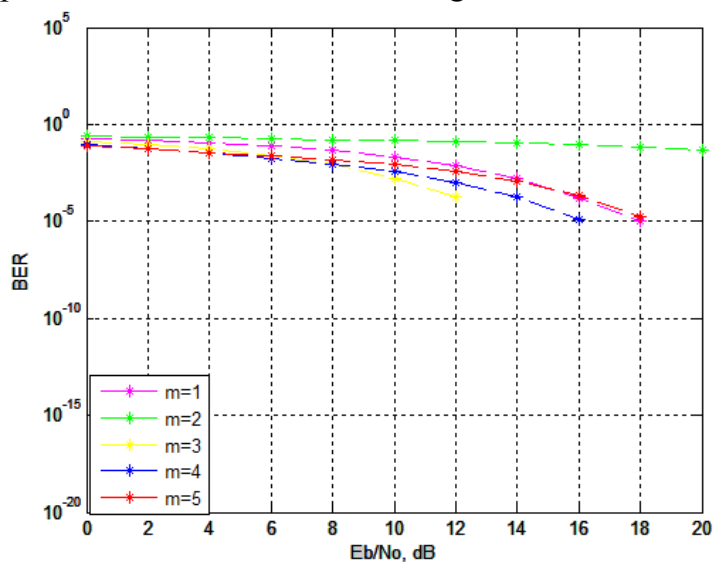

Fig. 10: Plot of computed channel BER vs. channel Eb/No for Clown with different fading parameter (m) and constant maximum shadowing parameter $(\mathrm{k}=1)$

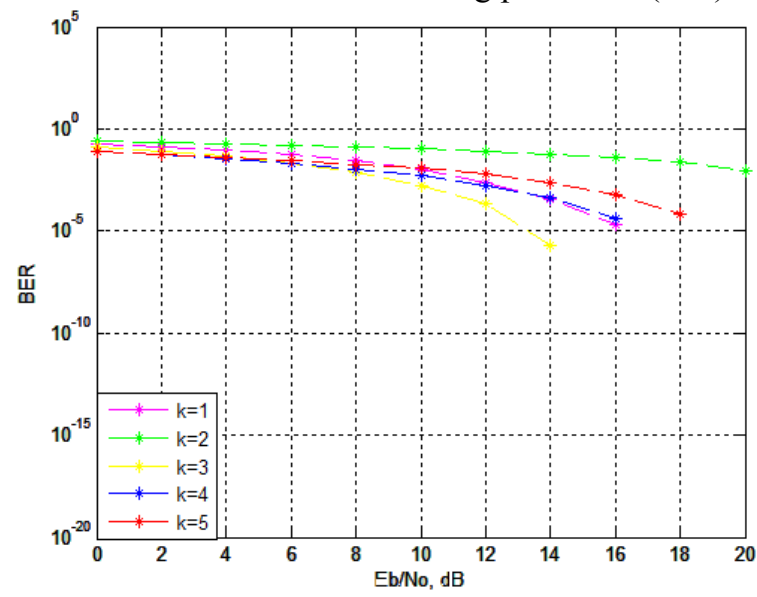

Fig. 11: Plot of computed channel BER vs. channel Eb/No for Clown with different shadowing parameter (k) and constant maximum fading parameter $(\mathrm{m}=1)$ 


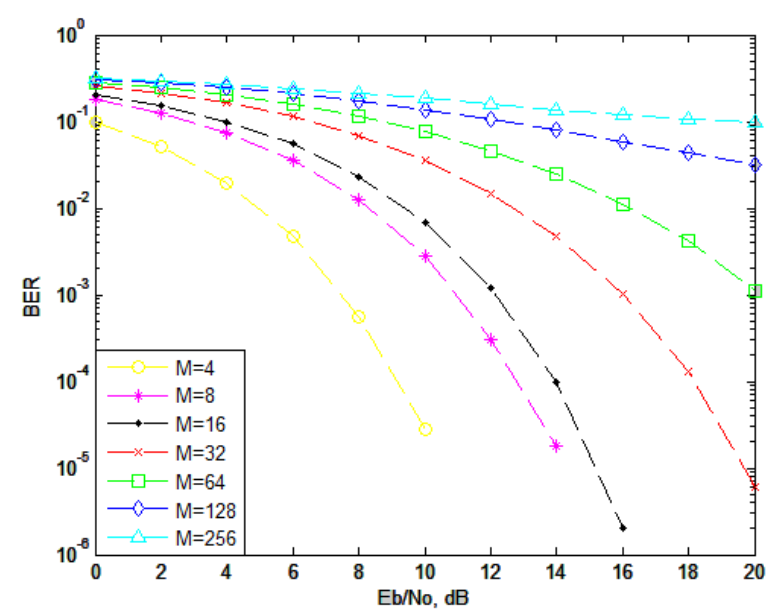

Fig. 12: Plot of computed channel BER vs. channel Eb/No for Clown with M-QAM

\section{Conclusion}

This paper successfully demonstrated the transmission of grayscale images using M-QAM OFDM System using Generalized K Fading channel. The Generalized K Fading channel is evaluated by transmitting still images through the 16-QAM modulated OFDM system using different fading parameters and Shadowing parameters values. The investigated results shows the effect of fading and Shadowing in terms of visual quality of received images and the bit error rate. The images being transmitted over the composite fading channels shows that they are exceptionally sensitive to effect of multipath propagation, which can deteriorate the quality of the image at the receiver. Since, the mobile radio signal envelope is usually composed of a fast fading component superimposed on a slow fading component, from the presented results it is clear that the channel model composed of mixed distributions is useful for designing wireless systems and networks

\section{References}

[1]. Lei Wan, V. K. Dubey, -Bit error probability of OFDM system over frequency nonselective fast Rayleigh fading channels, IEEE Electronics letters, Vo. 36, No. 15, pp1306- 1307, July 2000.

[2]. Jun Lu, Thiang Tjhung, Fumiyuki Adachi and Cheng Li Huang, -BER performance of OFDM-MDPSK system in Frequency Selective Rician Fading with Diversity Reception, IEEE Trans. On Vehicular Technology, vol. 49, no. 4, pp. 1216-1225, July 2000.

[3]. Yong Jae Ryu, Dong seong Han, -Timing phase estimator overcoming rayleigh fading for OFDM systems, IEEE Proc., pp. 66-67, 2001.

[4]. Mukesh Kumar Mishra, Neetu Sood and Ajay K Sharma. Article: BER Performance of OFDM-BPSK over Nakagami Fading Channels. International Journal of Computer Applications 17(8):1-3, March 2011.

[5]. Zhiqiang Liu, Georgios B. Giannaki, -Block Differentially Encoded OFDM With Maximum Multipath Diversity, IEEE Transactions on wireless communications, Vol.. 2, No. 3, pp. 420-423, May 2003.

[6]. M. Nakagami, - The m-distribution-A general formula of intensity distribution of rapid fading, in Statistical Methods in Radio Wave Propagation, W. C. Hoffman, Ed. Elmsford, NY: Pergamon, 1960

[7] Zhein gjiu Kang, Kung Yao, Flavio Lorenzelli, - Nakagami-m Fading Modeling in the Frequency Domain for OFDM system analysis, IEEE Communication letters, vol. 7, no.10, pp. 484-486, Oct.2003.

[8]. Zheng du,Julian Cheng and Norman c. Beaulieu, - Asymptotic BER performance of OFDM in Frequency Selective Nakagami-m Channels, IEEE Conference on Vehicular Technology, vol. 1, pp. 612-615,Sept. 2004.

[9] Zheng du, Julian Cheng and Norman c. Beaulieu, -Accurate Error Rate Performance Analysis of OFDM on Frequency Selective Nakagami-m Fading Channels, IEEE Trans.on communications.vol. 54, no. 2, pp. 319-328, Feb. 2006.

[10] Hui Liu and Guoqing Li ,"OFDM-Based Broadband Wireless Networks Design and Optimization”, John Wiley \& Sons, Mc. publication, 2005

[11] Shankar, P. M. "Error rates in generalized shadowed fading channels," Wireless Personal Communications, Vol. 28, No. 4, 233 238, Feb. 2004.

[12] Bithas, P.S.; Sagias, N.C.; Mathiopoulos, P.T.; Karagiannidis, G.K.; Rontogiannis, AA, "On the performance analysis of digital communications over generalized-K fading channels," Communications Letters, IEEE, vol.10, no.5, pp.353,355, May 2006

[13] William Stallings, Wireless Communications and Networking, Pearson Prentice Hall, 2005

[14] Gunjita Jain, Rupesh Dubey. "BER Performance of OFDM System over Nakagami - m Fading Channels with Different Modulation Schemes," International Journal of Advanced Research in Computer Engineering \& Technology (IJARCET), vol1, no.6, Aug 2012

[15] Dwivedi, V.K. and Singh, G. “ A NOVEL MGF BASED ANALYSIS OF CHANNEL CAPACITY OF GENERALIZED-K FADING WITH MAXIMAL-RATIO COMBINING DIVERSITY”, Progress In Electromagnetics Research C, Vol. 26, 153-165, 2012

[16] Danijela Aleksi, Mihajlo Stefanovi, Zoran Popovi, Dragan Radenkovi, Jovan D. Risti, "On the K and KG Fading Channels" SERBIAN JOURNAL OF ELECTRICAL ENGINEERING, Vol. 6, No. 1, May 2009, 187-201

[17] Bithas, P.S.; Sagias, N.C.; Mathiopoulos, P.T.; Karagiannidis, G.K.; Rontogiannis, AA, "On the performance analysis of digital communications over generalized-K fading channels," Communications Letters, IEEE, vol.10, no.5, pp.353,355, May 2006

[18] Shankar, P. M. "A Nakagami-N-gamma Model for Shadowed Fading Channels," Wireless Personal Communications, Vol. 64, No. 4, 665-680, June 2012.

[19] M.K. Simon, M.S. Alouini: Digital Communications over Fading Channels, John Wiley \& Sons, 2000

[20] P. Mohana Shankar, Fading and Shadowing in Wireless Systems, Springer, 2011 
[21] Yong Soo Cho, Jaekwon Kim, Won Young Yang, Chung G. Kang, MIMO-OFDM Wireless Communications with MATLAB, John Wiley \& Sons, 2010

[22] A. Dziri, M. Terre and N. Nasser, "Performance Analysis of Decode and Forward Cooperative Relaying over the Generalized-K Channel," Wireless Engineering and Technology, Vol. 4 No. 2, 2013, pp. 92-100. 